\title{
Usability Maturity model and Assessment Tool for Virtual Learning Systems
}

\author{
Kelvin K. Omieno ${ }^{1}$, Anthony Rodriguez ${ }^{2}$ \\ Lecturer, Department of Computer Science, Masinde Muliro University of Science \&Technology, Kakamega, Kenya ${ }^{1}$ \\ Professor, Department of Computer Science, School of Information and Innovative Systems, Jaramogi Oginga Odinga \\ University of Science \& Technology, Bondo, Kenya ${ }^{2}$
}

\begin{abstract}
Virtual Learning Systems (VLS) are becoming an increasingly common form of education due to the need for a platform that provides ability to connect people with required sets of skills, regardless of their location in the world. However, user satisfaction has been a major factor in the success of software, regardless of whether the software is proprietary or freeware. Although user-centred designs are gaining recognition among VLS community, many design scenarios still do not incorporate usability as one of their primary goals. Accordingly, many individuals believe that if VLS was more usable, its popularity would increase tremendously. Although there are strong usability models for information systems, there is still potential to improve the usability of virtual learning systems. The usability assessment of VLS is an area where relatively little research has been conducted, and, accordingly, the main contribution of this work is a tool that evaluates the usability maturity of a virtual learning systems.
\end{abstract}

Keywords: VLS, Usability Maturity, User-Centred Design, Metrics.

\section{INTRODUCTION}

VLS planform are becoming an increasingly common form of education due to the need for a platform that provides ability to connect people with required sets of skills, regardless of their location in the world. However, user satisfaction has been a major factor in the success of software, regardless of whether the software is proprietary or freeware. There is need for a well-structured assessment tool to measure usability of VLS. Usability Maturity Assessment Tool is a web-based system that is used to measure usability maturity levels in the use of virtual learning systems in public institutions. The system uses several metrics to come up with usability maturity level which were obtained from the research. The metrics used include User Requirements (UR), User Feedback (UF), Usability Learning (UL), UCD Methodology (UM), Understanding (UN), Learnability (LN), Operability (OP), Usability Bug Reporting (UBR), Usability Testing (UT) and Documentation (DOC). This chapter will provide Problem definition and justification, feasibility study, systems requirement and schedule.The origin of Usability Assessment Tool was founded on the research findings that identified the metrics and the parameters that give specific level of each parameter.

\section{PROBLEM DEFINITION}

The main goal of any university is to produce quality products through good teaching mechanisms that are customized to suit learners of different backgrounds and learning needs. Many universities are moving to the use of virtual learning systems to meet teaching needs of the learners[1][4]. However, the adoption of these systems and their use is largely dependent on their usability as indicated from the research. Usability of these systems was the primary concern of the research conducted. It is said that before you plan for your future, you need to know your situation. Consequently, it is important to know the level of usability of virtual learning systems so that the universities can find ways to improve their usability thus improving their adoption. It was discovered that there was no tool in existence that can be used to measure usability of virtual learning systems.

\section{METHOD}

The study also sought to determine the key factors used in presenting the various measures in the usability maturity assessment model for assessing usability of the VLS; and propose possible measures for VLS-Usability Maturity Assessment tool for universities. To achieve this purpose, exploratory research design was used to determine the various factors guiding design of VLS [2]. Data was collected using questionnaires and analyzed using SPSS Ver.20.The development of the system was based on Incremental Model of software development. The system was broken down into modules or subtasks.

\section{VLS USABILITY MATURITY MODEL}

In comparison to other aspects of software, usability issues are more subjective in nature and hence more controversial. In some instances, a user interface (UI) element may be clearer to some users than it is to others [3]. This is not different in VLS systems. However, 
Vol. 5, Issue 12, December 2016

despite their subjective nature, usability aspects need to be key usability factors. After combining several overlapping tested and measured objectively. The increasing popularity factors, such as User Requirements and User Expectations, of usability assessment in virtual learning systems Incremental Design Approach, Design Techniques and necessitates a usability maturity evaluation Knowledge of UCD Methods, and Usability Testing and methodology.From the empirical study from VLS users Usability Assessment, ten (10) key usability practices (the students and staff), the study has been able to identify were obtained, which are depicted in Table 1.

\section{TABLE 1 VLS ASSESSMENT METRICS}

\begin{tabular}{|l|l|c|l|}
\hline Dimension No. & VLS Usability Assessment Metrics & Question No. & Key Usability Factors \\
\hline \multirow{2}{*}{} & Usability Methodology & 1 & User's Requirements \\
\cline { 3 - 4 } & & 2 & User's Feedback \\
\cline { 3 - 4 } & & 3 & Usability Learning \\
\hline \multirow{2}{*}{2} & Design Strategy & 4 & UCD Methodology \\
\cline { 2 - 4 } & & 5 & Understandability \\
\cline { 2 - 4 } & & 6 & Learnability \\
\cline { 2 - 4 } & & 7 & Operability \\
\hline 3 & Assessment & 8 & Usability Bug Reporting \\
\hline 4 & & 9 & Usability Testing \\
\hline & Documentation & 10 & Documentation \\
\hline
\end{tabular}

It is necessary to determine the contribution of each of the regression analyses. A multiple regression method uses usability maturity assessment quality factors which correlation between a dependent variable and independent includes user's requirements, user's feedback, usability variables as a criterion to determine which variables would learning, User-Centred design methodology, learnability, be included in the regression model. As such, potential operability, usability Bug reporting, Usability Testing and variables for the regression model had to be correlated. To Documentation to determine their contribution to overall test the potential of the total scores for the ten dimensions, usability maturity assessment framework. For every a correlation matrix was computed and presented in the respondent, a total score for each dimension was Fig 2 below.

calculated. These total scores were used in multiple

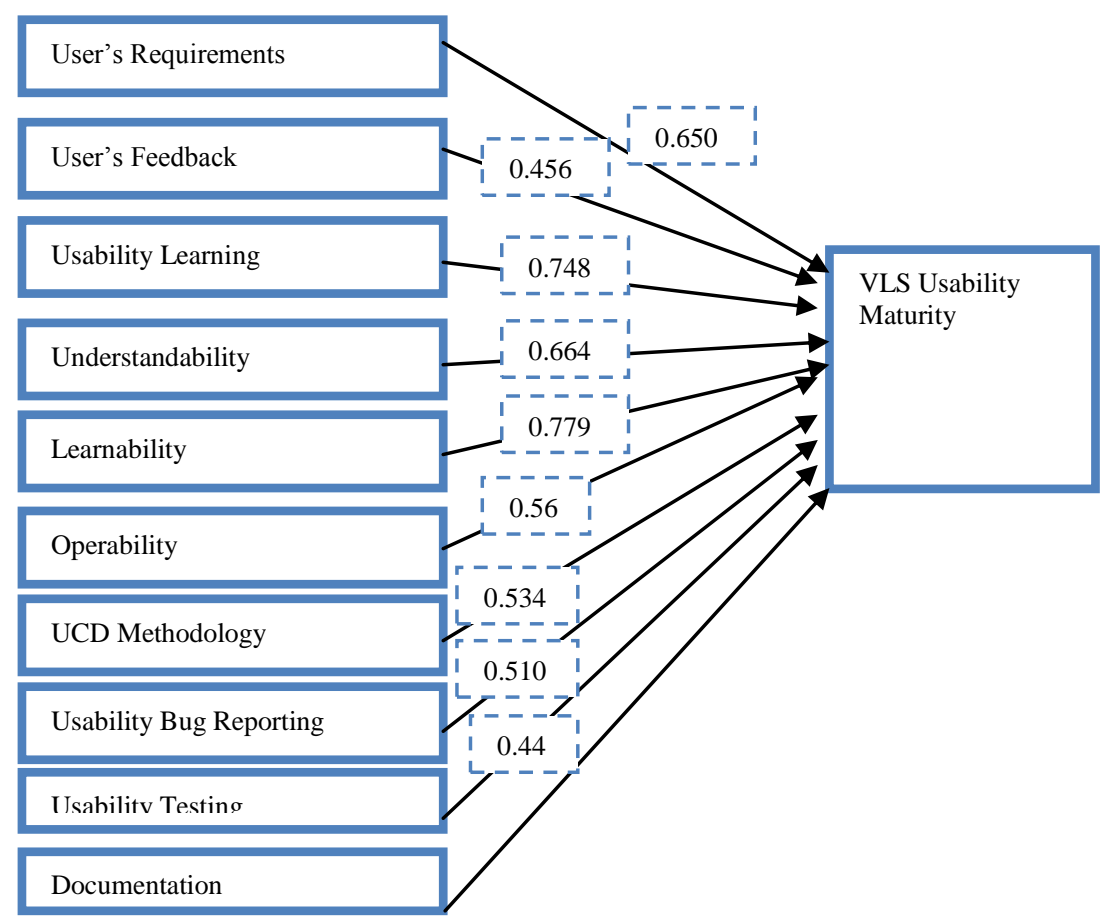

Figure 2: Weighing of Usability Maturity Matrix

VLS Maturity Model heavily lies in Software Engineering Design Strategy (DS), Usability Assessment (UA) and domain [6] and here we define four categories of Documentation (D). The visual summary of the model is assessment metrics namely: Usability Methodology (UM), summarized in the Fig. 10.In thinking about the 
Vol. 5, Issue 12, December 2016

relationship between the four dimensions it is helpful to within or between institutions but it can imply a consider them arranged as in Fig.3 below. The matrix of hierarchical relationship that is misleading when boxes used on the left to display summaries of process interpreting individual process maturity results. maturity metrics is helpful when performing comparisons

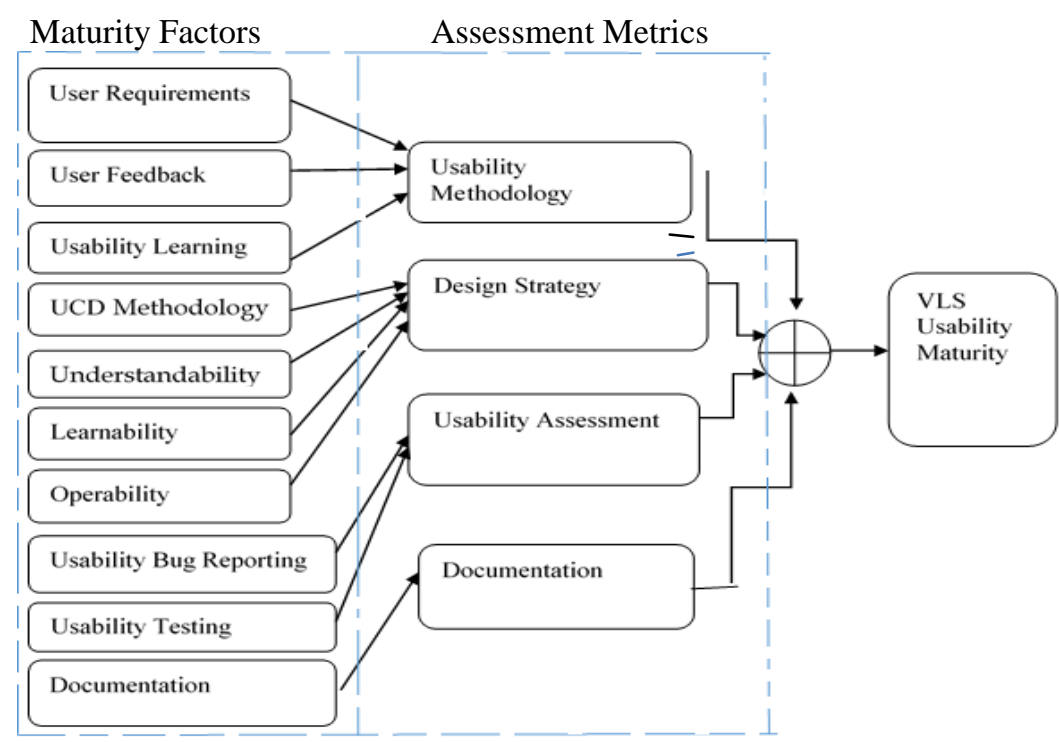

Figure 3. VLS-Usability Maturity Model

\section{DESCRIPTION OF LEVELS FOR USABILITY MATURITY ASSESSMENT- HUMAN CENTEREDNESS SCALE}

The usability maturity of VLS, in universities, is determined by the extent to which the content developers (who are teachers) and student (learners) agree with each statement in the questionnaire. The number of statements varies for each maturity level as well as for each usability factor.

\section{A. Level 1: Un recognized}

Indicates that the VLS system does not have a stable and organized methodology for implementing usability. The need for a human-centred process is not recognised. If systems are received with varying degrees of satisfaction by their end users this does not cause concern. There are no positive human-centred attributes at this level. There is no evidence that the VLS team practices usability to improve the software quality of their system. Additionally, there are no defined procedures for collecting user requirements and feedback, implementing usability learning, incorporating user design methodology, performing usability assessment and providing documentation.

\section{B. Level 2: Recognized}

University recognises that there is a need to improve the quality in use of its systems. The organisation has a development process and produces systems. Members of the organisation understand the business benefit of producing usable products such as VLS. Users recognize the potential benefits of usability in VLS and accordingly, they demonstrate interest in usability. Also, users make efforts to collect user requirements and feedback, and present the same to developers where the developers acquire knowledge of UCD methodology. Both end-users (students and staff) and developers recognize the fact that end users face difficulties in reporting usability related errors and hence understand the need for usability assessment plans. Additionally, the content developers (who in this case are staff) realize the importance of documentation at various stages of VLS content understand and recognize the importance of usability in the success of the system

\section{Level 3: Defined}

A VLS system at the "Defined" level establishes an infrastructure for implementing usability. Specifically, VLS team is able to collect and fulfil its users' requirements and expectations. Furthermore, they are able to collect feedback from users by utilizing a planned strategy for improving both software quality and usability. It is conceived that VLS managers understand, define and implement user-centered design principles. Through a systemic monitoring structure, team members (inclusive of the users) have the competency to maintain and enhance a project's satisfaction, understandability, learnability, operability and security. The team also adopts essential technical skills to provide users with a convenient usability bug reporting facility and to conduct necessary usability testing.

D. Level 4: Streamlined

Human-centred processes are integrated into the quality process and systems life cycle of the university. The 
Vol. 5, Issue 12, December 2016

systems and human-centred life cycles are managed to ensure that the results of the human-centred processes produce improvements in all relevant work products. The VLS team (especially the VLS providers) has acquired sufficient resources for meeting its users' requirements. Additionally, they have established a management system for recording users' feedback and taking the necessary steps to address that feedback. The users too are much aware of the steps too. Through a systemic monitoring structure, satisfaction, understandability, learnability, operability and security of VLS are regularly monitored.Moreover, quantifiable metrics are used to conduct usability assessments, and the documentation of VLS projects is regularly maintained.

\section{E. Level 5: Institutionalized}

For virtual learning systems, the highest level for the usability maturity is referred to as "Institutionalized." The quality in use of whole ranges of VLS systems is coordinated and managed for business benefit. The culture of the organisation gains benefit from being user and human centred. Human-centred processes are used within the organisation to improve the quality in use of the processes, tools and methods used and developed by the organisation for its own use. Specifically, the VLS team has sufficient resources and skills for collecting user feedback and for understanding user expectations. Similarly, the team establishes a firm commitment to usability learning and UCD methodology, and they continuously make Assessments in the areas of satisfaction, understandability, learnability, security and operability and they constantly improve the usability bug reporting service through innovative methods and ensure the effectiveness of usability assessment by using quantitative metrics.

\section{VLS DESIGN ISSUES AND DEVELOPMENT}

\section{A. VLS performance scale}

The maturity level of a VLS is determined by its ability to demonstrate the usability factors for that particular level. In order to ascertain maturity level, five-point scale have been used to rate a project's performance. The ordinal ratings used to measure each usability factor, including "Fulfilled", "Largely Fulfilled", "Partially Fulfilled", "Not Fulfilled" and "Not Applicable", are borrowed from BOOTSTRAP methodology by Wang \& King. [5], and are shown in Table2

\section{TABLE 2 VLS PERFORMANCE SCALE}

\begin{tabular}{|l|l|l|l|}
\hline Scale & \multicolumn{2}{|l|}{$\begin{array}{l}\text { Expression of } \\
\text { Performance in English }\end{array}$} & $\begin{array}{l}\text { Rating } \\
\text { threshold (\%) }\end{array}$ \\
\hline $\mathbf{0}$ & VLS-UMM & BOOTSRAP & \\
\hline $\begin{array}{l}\text { Not- } \\
\text { applicable }\end{array}$ & - & - \\
\hline $\mathbf{1}$ & $\begin{array}{l}\text { Not- } \\
\text { fulfilled }\end{array}$ & Absent /Poor & $<=33.2$ \\
\hline
\end{tabular}

\begin{tabular}{|l|l|l|l|}
\hline $\mathbf{2}$ & $\begin{array}{l}\text { Partially } \\
\text { fulfilled }\end{array}$ & $\begin{array}{l}\text { Partially } \\
\text { Satisfied }\end{array}$ & $33.3-66.6$ \\
\hline $\mathbf{3}$ & $\begin{array}{l}\text { Largely } \\
\text { fulfilled }\end{array}$ & $\begin{array}{l}\text { Largely } \\
\text { satisfied }\end{array}$ & $66.7-79.9$ \\
\hline $\mathbf{4}$ & Fulfilled & $\begin{array}{l}\text { Completely } \\
\text { satisfied }\end{array}$ & $>=80$ \\
\hline
\end{tabular}

B. VLSRating Methodology

In order to derive the rating methodology for the proposed Usability Maturity Assessment Tool, the proposed rating methodology is partially derived from the BOOTSTRAP rating methodology as earlier observed (Wang and King 2000). Borrowing concepts from the algorithm, the study has adopted terms, such as VLS Usability Rating (URv), Number of Fulfilled Statements (NF), Passing Threshold (PT) and Usability Maturity Level (UML). In this study, let URv [i,j] be a rating of the ith usability factor of the jth maturity level. Subsequently, according to the scales for VLS performance defined in Table 5.2, it can be summarized as:

$\mathrm{URv}[\mathrm{i}, \mathrm{j}]=4$, if the fulfillment of the condition or the statement is at least $80 \%$

$=3$, if the fulfillment of the condition or the statement is from 66.7 to $79.9 \%$

$=2$, if the fulfillment of the condition or the statement is from 33.3 to $66.6 \%$

$=1$, if the fulfillment of the condition or the statement is less than $33.3 \%$

$=0$, if the condition or the statement is not applicable.

Then, the observation $i^{\text {th }}$ condition/statement at the $j^{\text {th }}$ maturity level is considered fulfilled if $\operatorname{URv}[i, j] \geq 3$ or URv $[i, j]$ is 0 . The number of conditions/statements fulfilled at jth maturity level is defined as:

$\mathrm{NF}[\mathrm{j}]=$ Number of $\{\mathrm{URv}[\mathrm{i}, \mathrm{j}] \mid$ Fulfilled $\}$

$=$ Number of $\{U R v[i, j] \mid U R v[i, j] \geq 3$ or

$[\mathrm{i}, \mathrm{j}]=0\}$

There is however an observation: that the usability maturity is considered to be achieved if $80 \%$ of the conditions or statements in the questionnaire are fulfilled. Thus, if $\mathrm{TN}[\mathrm{j}]$ is the total number of statements at the jth maturity level, then the passing threshold at the jth maturity level is defined as:

$$
\mathrm{PT}[\mathrm{j}]=\mathrm{TN}[\mathrm{j}] * 80 \%
$$

In this context, the Usability Maturity Level (UML) is defined as the highest maturity level at which the number of fulfilled conditions or statements is greater than or equal to the passing threshold PT [j] hence:

$$
\mathrm{UML}=\max \{\mathrm{j} \mid \mathrm{NF}[\mathrm{j}] \geq \mathrm{PT}[\mathrm{j}]\}(3)
$$

Where max represents a function of Number of URv fulfilled at $\mathrm{j}^{\text {th }}$ maturity level

\section{Architecture of the VLS Tool}

Since the target users of the model were widely dispersed geographically, a web based platform was considered the most appropriate. The client/server architecture base on 3tier was therefore considered to be the most suitable to implement the tool. The following open-source tools were selected for each tier: 
i. Client side scripting: HTML5 and Java script

ii. Server side scripting: PHP

iii. Database: MySql

Figure 1 presents general overview of the Design of the UMM_VLS tool.

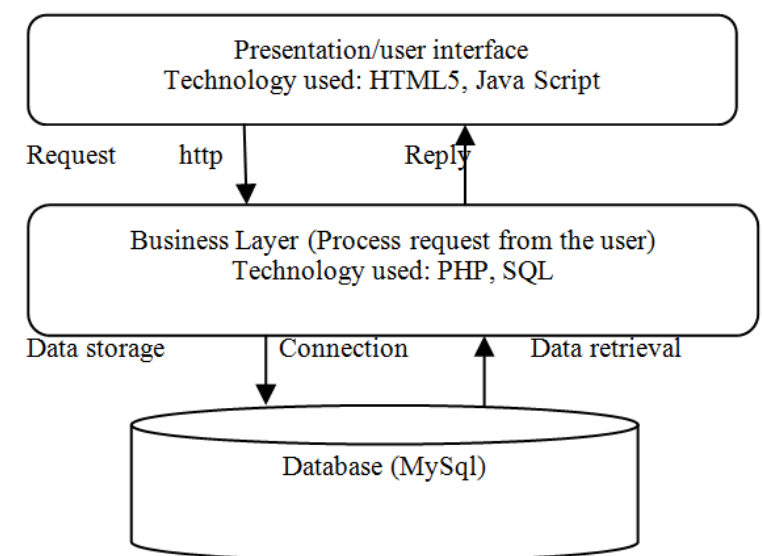

The back end of the system consists of the MySQL database that is used as a storage media for data entries (made from the system interface) and PHP Parser. The user is not aware of this as she/her just interacts with the system interface provided for them in the front end. The front end is the system interface that the user interacts with in order to achieve its functionality. It is made possible by using web browsers e.g. Mozilla Firefox or Internet explorer.

D. Architectural Design

Software architecture is often described as the organization or structure of a system, where the system represents a collection of components that accomplish a specific function or set of functions. In other words, architecture is focused on organizing components to support specific functionality. In the present study, the architectural design of the UMM_VLS tool is as described in the Figure 5.

Figure 4: High level design view of the VLS-UMM tool

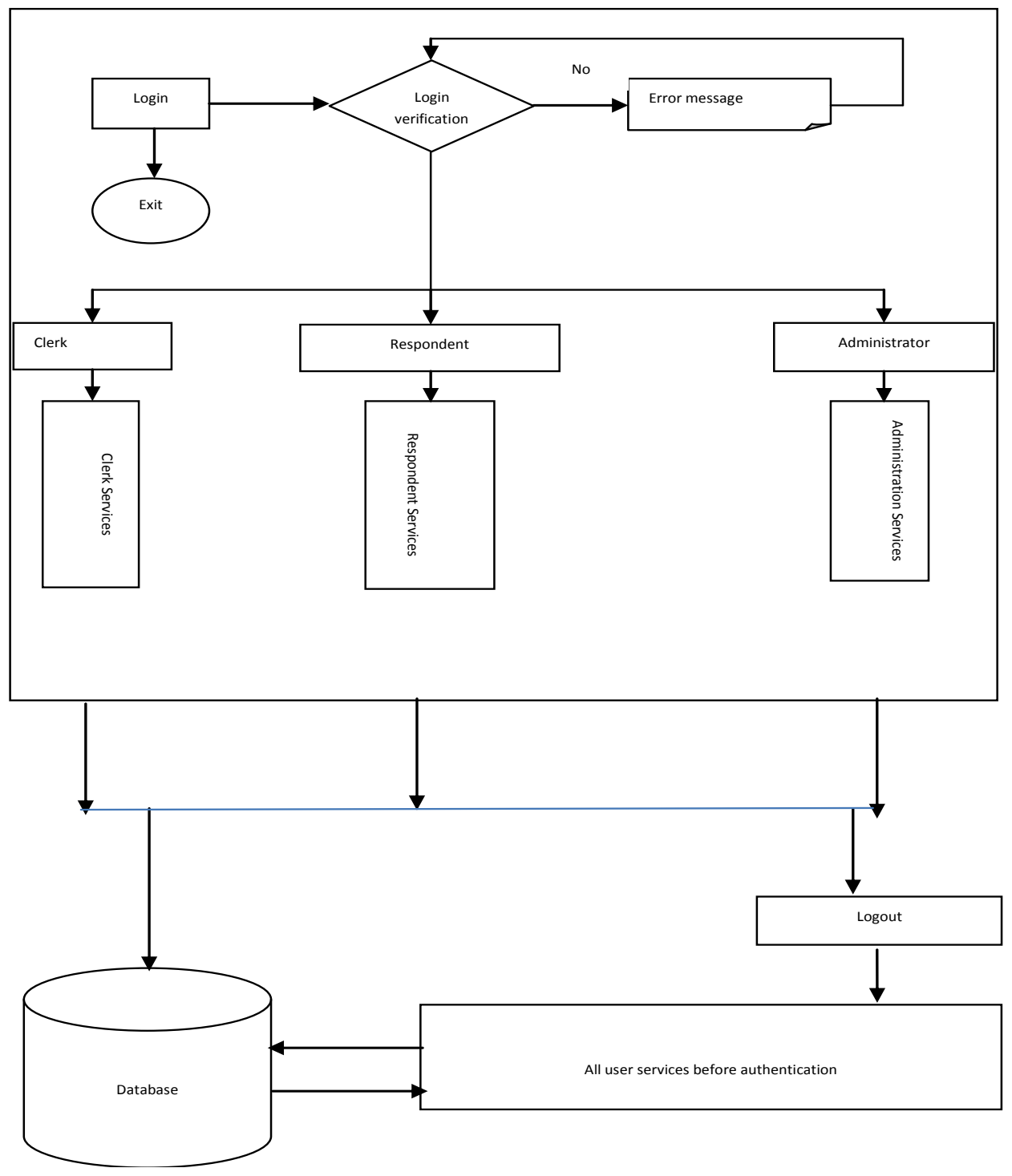


E. Abstract Interface Design

The abstract interface of UMM_VLS tool is an interface containing Links to various functionalities to the Top and to the Left of the system interface for analysis window. The functionalities of the system are classified into three main categories: Respondent services, Data Entry Clerk services and Administrator services. Respondent services are accessed by every user who wants to measure usability maturity of a given virtual learning systems. All users are authenticated using username and password that meets a given strength. If the authentication succeeds, the user is allowed to access requested services. If the authentication fails, the error message is issued and the user is given a chance to Login as illustrated in Fig.6 below.

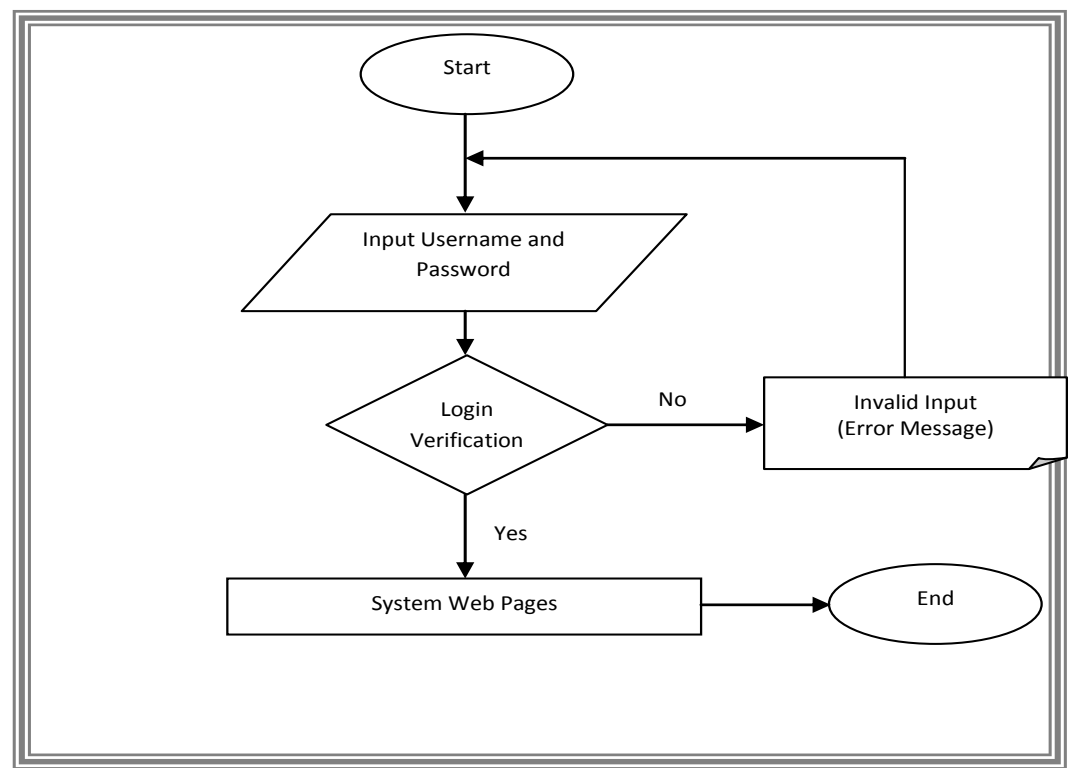

Figure 6: High level design view of the VLS-UMM tool

F. Design of System Back-end

\section{i. View Schema}

The data structure of the system is based on the objects which translate to real world entities which are the building blocks of UMM_VLS Tool. The entities identified in conceptual model of the system database include: -

a) Metric

b) Question

c) Response

d) Users

The associated attributes identified were:-

Metric: \{ Metric Code, Metric Name, Description, Priority Options \}

Institution: \{ Institution Code, Institution Name, Description, Postal Address, Email Address, Phone Number, Telephone Number, Fax

Question: \{ Question Code, Metric Code, Description, Level, Priority Options \}

Response: \{ Response Code, Question Code, Response, Institution Code

User: \{ User ID, Login Name, Short Name, Long Name, Postal Address, Email Address, Phone number, Telephone number, Privileges, Institution Code, Password, Status \}

\section{ii. Logical Model}

The logical model of the system database resulted from the identified entities which led to formation of five tables with their relationship with one another. The individual relationships are identified and the Entity Relationship Diagram is given also shown.

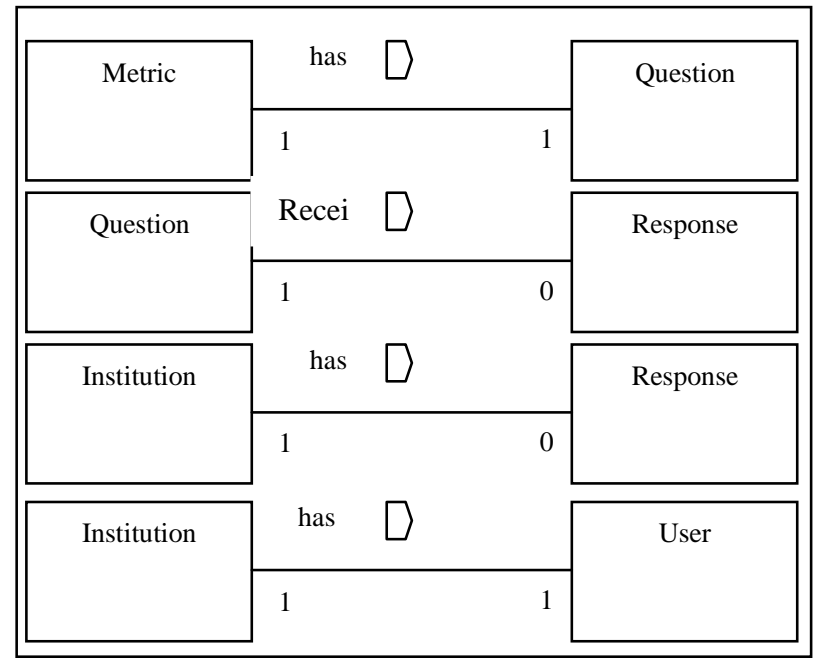

Figure 7: Identified Relationships

\section{iii. Physical Schema}

The actual database was created using Data Definition Language (DDL) statements.

Data definition language (DDL) statements was specifically useful in performing varied tasks including: create, alter, and drop schema objects; grant and revoke 
Vol. 5, Issue 12, December 2016

privileges and roles; analyze information on a table, index, or cluster; establish auditing options; and adding comments to the data dictionary. This is as illustrated in Appendix J.

\section{G. System Implementation \\ i. $\quad$ Coding}

After finishing the designing of the system, the code of the system was written. The system database was first developed using MySQL commands. After that, the system's Graphical User Interface (GUI) was developed using PHP by the help of Adobe Dreamweaver CS4/CS5. To accomplish this, the system GUI was divided into modules and developed one module after the other.

After developing the first module, the other modules were rather easier to develop because it was more like a repetition. It is worth mentioning that the whole system interface was developed using Adobe Dreamweaver CS4/CS5 and hard coding processes. Sample code is given at the end of this report that displays the Usability maturity level (Appendix I).

\section{ii. Module Integration and Testing}

Module integration was done in parallel with coding processes since to realize various functionalities of a module; one has to integrate with another module. After developing all modules, they were integrated together by the help of links which made moving from one module to another possible. The connection between web pages and the database was done by creating a connection in the Adobe Dreamweaver CS4/CS5 interface which led to insertion and reading of values from the database.

The module verification was carried out at the end of each module to check if it is constructed as expected and evaluation to check if the module works as expected- unit testing. After completely developing the applications modules, the modules were linked and tested if they can work together.

During this integration testing, inter-module conflicts that were spotted were resolved and ensured that different modules work well together to achieve the overall goal of the system

\section{H. Execution of the VLS tool}

The user is presented with a login/register page where they are required to register if they are first time users or login if already registered. When the user loads into the system address, the home page (shown in Fig. 8) of the system appears providing links to functionalities.

These functionalities are accessed by selecting them from horizontal menu. Some of the menus available are Home that takes a user to Home page of the system, Design which provides submenus for managing metric questions and institutions, Response that has submenus for giving response and analysis, Administration menu for managing users, Contact us and Help.

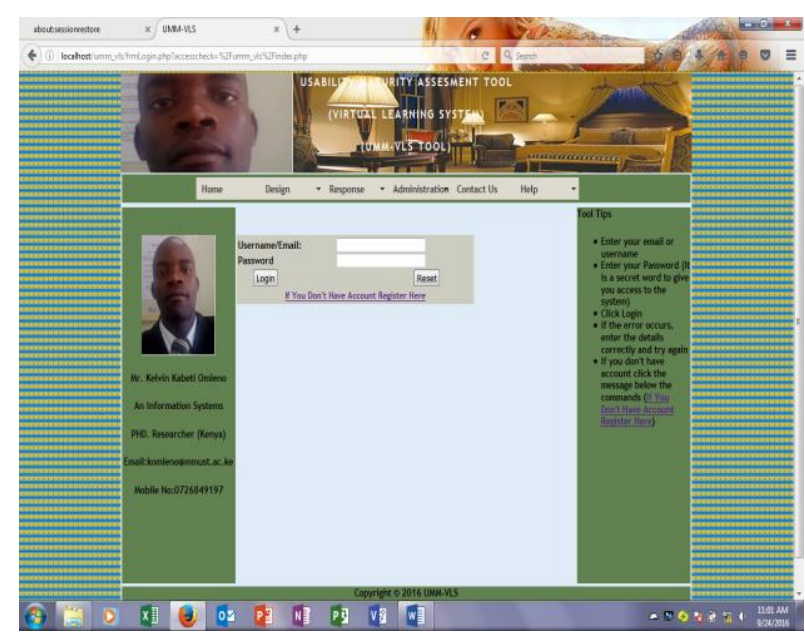

Figure 8: Login/Registration interface of VLS-UMM tool

Once logged in, the user is presented with a home page from where they can register institution by clicking Design->Institution menu as depicted in Figure 9. The same applies to design of VLS Metric, Question and Institution as submenus as illustrated in Fig 9

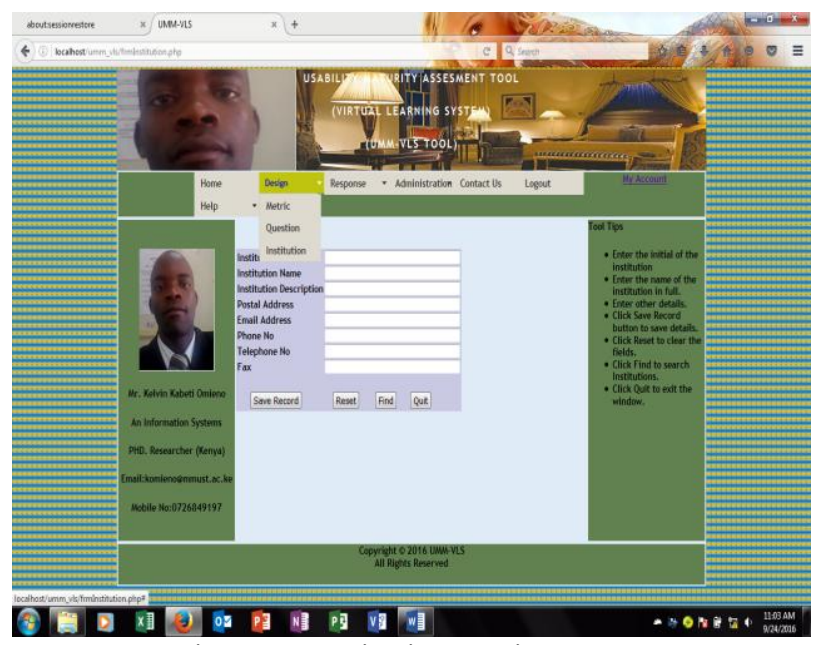

Figure 9. Institution Design menu

The final analysis of the responses is obtained by following Response menu which basically offers two submenus i.e. Response and Analysis. Response offers an interface to the user to respond on the questionnaires on each metric.

A user is expected to save response in one page before moving to the next page. A user is provided a question that requires one response from the respondents. The response is supposed to be within five options which include Strongly Agree, Agree, Neutral, Disagree and Strongly Disagree as shown in Fig. 10 show below.

The analysis option presents the user with analysis report window that will allow users to obtain reports on Respondents, Raw Response, Metric Response, Response Summary and UML. The UML window is shown for all universities entered as depicted in Fig 11. 
Vol. 5, Issue 12, December 2016

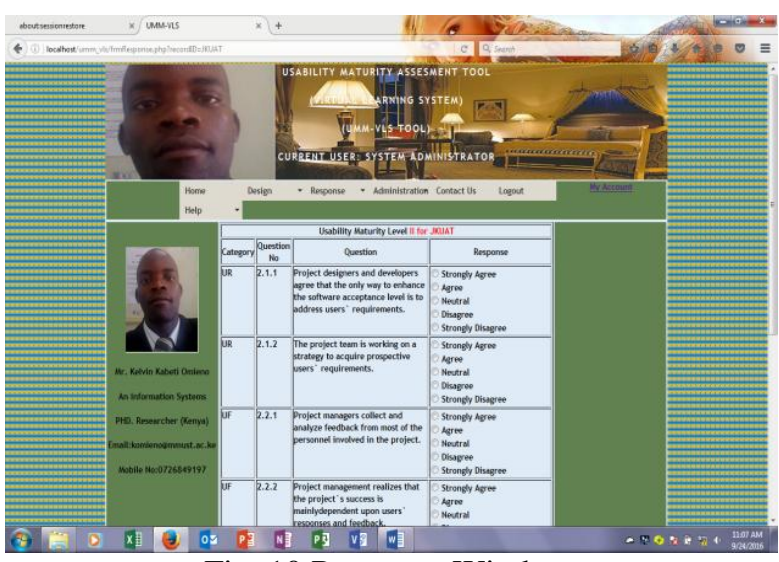

Fig. 10.Response Window

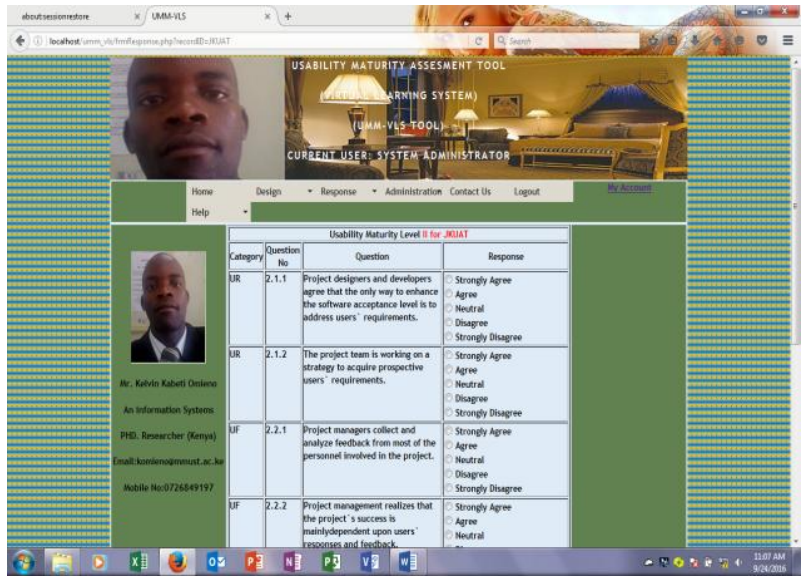

Figure 11: Sample output of analysis window of the VLS tool

\section{CONCLUSION}

The paper presents factors used in presenting the various measures in the usability maturity assessment model for assessing usability of the VLS; and presents and VLSUsability Maturity Assessment tool for Kenyan universities. It was observed from the research findings that experiences of end users have a profound impact on the success of a software project, and, as a result, usability and its related issues are a key area of research in virtual learning systems. The research showed that this situation is largely because of poor usability of these virtual learning systems and therefore, there was need to come up with an automated tool to measure usability maturity for virtual learning systems that will help them improve their learning systems and consequently increasing their adoption. A few of the challenging options for improving VLS usability include understanding users' expectations through empirical investigations, adapting approaches in VLS designs to improve usability and quantifying usability metrics. However, in order to identify the precise areas where improvement is necessary, assessment needs to be performed. Due to a continual increase in the number of both technical and non-technical users (who form the student and staff using the e-learning platform), the evaluation of VLS usability requires a comprehensive strategy, which has not yet been fully explored. Unfortunately, the adoption of virtual learning systems has not been that impressive.

\section{REFERENCES}

[1] Millward, L. J., \& Kyriakidou, O. . Effective Virtual Teamwork: A Socio-Cognitive and Motivational Model. In S. H. Godar \& S. P. Ferris (Eds.), Virtual and Collaborative Teams: Process, Technologies and Practice (pp. 20 34): Idea Group Publishing, 2004

[2] Creswell, J. W. Research design: Qualitative, quantitative and mixed method approaches. Thousand Oaks, CA: Sage, 2014.

[3] Gulliksen, J., B. Goransson, I. Boivie, S. Blomkvist, J. Persson, and A. Cajander. Key Principles for User-Centred Systems Design. Behaviour and Information Technology 22, 397-409, 2003

[4] Mitchell, A., \& Zigurs, I. Trust in Virtual Teams: Solved or Still a Mystery? The DATA BASE for Advances in Information Systems, 40(3), 61-83, 2009

[5] Seffah, A; Donyaee, M \& Kline, R.. Usability measurement and metrics: A consolidated model. Software Qual J 14: 159-178, 2006

[6] Sauser, B; and Ramirez-Marquez, J. Development of Systems Engineering Maturity Models and Management Tools, Report No. SERC-2011-TR-014, 2011

\section{BIOGRAPHIES}

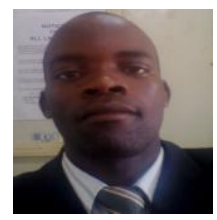

Kelvin Omieno has completed his $\mathrm{PhD}$ in Information Systems and is a Lecturer ar Masinde Muliro University of Science and Technology. He has research interests in the area of learning information systems and cloud-based computing. He is a professional member with Association of Computing Machinery.

Prof. Anthony Rodriguez is a professor in Computer Science. He is widely read and has international standing in the field. He is a member of several professional bodies and a reviewer with a number of journals. He is currently as director of ICT at Jaramogi Oginga Odinga University of Science and Technology.

\section{APPENDIX I}

\section{$<$}

?php do \{ \$_SESSION['VLS']="NA";?>

$<\operatorname{tr}>$

$<$ td $\quad$ valign="top" $><$ ?php echo

\$row_rsGetValues['Institution_Code']; ? $>\langle/$ td $\rangle$

$<$ td $><$ ?php if(\$row_rsGetValues['Institution_Code'] $<>"$ ") \{

if(\$row_rsGetValues['Level_1_Per'] $>0$

$\& \&$

\$row_rsGetValues['Level_1_Per']<=33.2)

\section{\$_SESSION ['VLS']="NF";}

\}

elseif(\$row_rsGetValues['Level_1_Per']>33.2

$\& \&$ \$row_rsGetValues['Level_1_Per']<=66.6)

\{

\} 
elseif(\$row_rsGetValues['Level_5_Per']>66.7

$\& \&$ \$row_rsGetValues['Level_5_Per'] $<=79.9$ )

\{

\}

$$
\text { \$_SESSION['VLS']="LF"; }
$$

elseif(\$row_rsGetValues['Level_5_Per']>79.9

$\& \&$ \$row_rsGetValues['Level_5_Per']<=100)

$$
\{
$$$$
\text { \$_SESSION['VLS']="FF"; }
$$

$$
\text { \} }
$$

else

$$
\text { \{ }
$$

$$
\text { \$_SESSION['VLS']="NA"; }
$$

echo

nl2br(\$row_rsGetValues['Level_5']."In".round(\$row_rsGe tValues['Level_5_Per'],2).'\%'."'In".\$_SESSION['VLS']); $?>\langle/$ td $>$

$<$ td $><$ ?php if(\$row_rsGetValues['Institution_Code'] $<>"$ ")

$$
\{
$$

if(\$row_rsGetValues['Average_Response_Per']<

$$
\text { \} } \quad \text { \$_SESSION['VLS']="NF"; }
$$

elseif(\$row_rsGetValues['Average_Response_Pe r'] $>33.2$ $\& \&$ \$row_rsGetValues['Average_Response_Per']<=66.6) \{

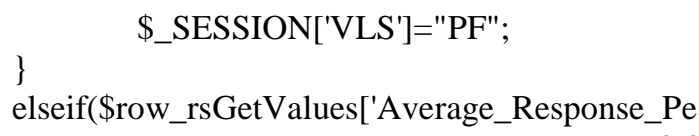

nl2br(\$row_rsGetValues['Average_Response']."In".round( \$row_rsGetValues['Average_Response_Per'],2).'\%'."In".\$ _SESSION['VLS']);\} ?></td $>$

$</ \mathrm{tr}>$

$<$ ?php while (\$row_rsGetValues = mysql_fetch_assoc(\$rsGetValues)); ?> 